\title{
Klappern gehört zum Handwerk!
}

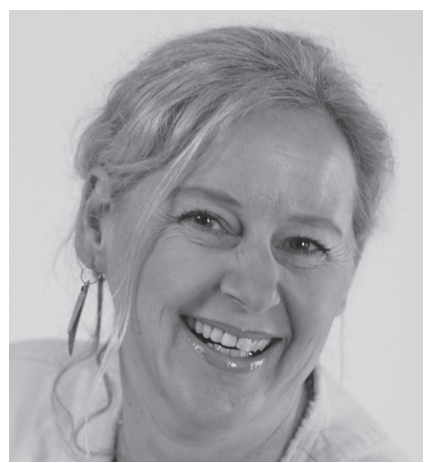

VON MARTINA PETERS

Martina Peters arbeitet als freie Journalistin und Trainerin mit den Schwerpunkten Bildung, Presseund Öffentlichkeitsarbeit und Fundraising. In ihren Seminaren geht es beispielsweise um Themen wie "Meckern als Chance - Antwortbriefe auf Beschwerden als PR nutzen«, »Besser schreiben« und "Mitarbeiterzeitung". www.martina-peters.de

\author{
Fundraising und Öffentlichkeitsarbeit liegen \\ nah beieinander. Um sich mit guten Projekten \\ publikumswirksam präsentieren zu können, sind \\ soziale Organisationen heutzutage häufig auf Geld \\ und Sachleistungen von Förderern und Sponsoren \\ angewiesen. Soziale Dienste und Verbände müssen \\ sich deshalb mit einem besonderen Profil einen Namen \\ machen, wenn sie sich gegen die Politik behaupten, \\ in der Öffentlichkeit ein gutes Image erlangen und \\ zusätzlich noch Förderer gewinnen möchten.
}

Öffentlichkeitsarbeit und Fundraising für Non-Profit-Organisationen? Die erste Reaktion von Verantwortlichen insbesondere kleiner Träger ist häufig: Wann sollen wir das denn noch machen? Verständlich, denn Soziale Arbeit, Organisation, Dokumentation, Arbeit mit Ehrenamtlichen und andere Aufgaben nehmen schon genug Zeit in Anspruch. Aber der Aufwand lohnt sich. Denn wenn Public Relations (PR) und Fundraising gut gemacht werden, das heißt mit Konzept geplant sind und professi- mit Sponsorengeldern finanzieren? Wie wäre es mit einer Imagebroschüre? Eintagsfliegen, sporadische Anstrengungen und halbherzige Schnellschüsse wegen Zeitmangel haben allerdings keinen nachhaltigen Effekt. Wie das Kerngeschäft der Organisation, die Soziale Arbeit, sollten auch Öffentlichkeitsarbeit und Fundraising gut geplant werden.

Presse- und Öffentlichkeitsarbeit von Non-Profit-Organisationen vermag viel. Sie kann die unterschiedlichsten, für soziale Träger relevanten Zielgruppen

\section{"Auch wer nichts sagt, sendet eine Botschaft"}

onell umgesetzt werden, können sie viel bewirken und die fachliche Arbeit von sozialen Diensten und Einrichtungen nachhaltig verbessern.

Irgendwann kommt fast jeder Verantwortliche an den Punkt, an dem sich Fragen wie diese stellen: Sollten wir nicht auch die Presse zum Tag der offenen Tür einladen? Vielleicht können wir den Kaffeeautomat für die Tagesstätte informieren, positiv beeinflussen und überzeugen. Sie kann das Selbstbewusstsein des Teams und der Mitglieder sowie die inner- und außerorganisatorische Kommunikation und damit die Position im Wettbewerb stärken. Und sie kann das Verständnis der Öffentlichkeit in Krisensituationen, die Transparenz und das Organisationsklima, ein schlechtes Image sowie die Netzwerkarbeit im 


\section{Checkliste PR-Konzept}

Das Konzept für Public Relations (PR) umfasst folgende Aufgaben und Fragestellungen:

\section{Analyse}

- Kurzprofil erstellen: Wer sind wir? Wie können wir unsere Corporate Identity, unser Organisationsprofil in drei Sätzen beschreiben? Also: Was macht uns als sozialer Träger aus? Dazu gehört das Dienstleistungsangebot, das Leitbild, das äußere Erscheinungsbild, die Kommunikation nach innen und außen, die Regeln, die Organisationsphilosophie usw.

- Beschreibung der Ausgangslage und Imageanalyse: Was haben wir zu bieten? Welche Ausrichtung hat unsere Organisation (politisch, religiös, humanistisch, anthroposophisch o. Ä.) Welches Image haben wir im Stadtteil, in der Stadt?

- Stärken-Schwächen-Analyse: Was läuft gut, was läuft schlecht? Stimmung im Team, Organisation des Angebots, Informationsfluss, Motivation etc.

2. Ziel(e)

- Was wollen wir erreichen? Drei Zeitungsartikel bis Weihnachten? Mindestens eine Ausstellung in der Tagesstätte? Mindestens einen Förderer für die Hausaufgabenhilfe? Eine präzise, konkrete Zielformulierung erleichtert die Evaluation.

- Formulierung von Botschaften und Ideen: Was wollen wir in die Öffentlichkeit tragen? Beispiele. Unser ambulanter Dienst legt besonders viel Wert auf die Einbeziehung der Angehörigen und des Umfelds. Oder: Unsere Verein

Stadtteil verbessern.

Wenn eine Organisation das Thema Öffentlichkeitsarbeit gezielt angehen möchte, hat sich folgendes Vorgehen bewährt: Zum Auftakt sollten sich ein paar engagierte und kreative Kolleginnen und Kollegen treffen und zunächst einmal »gucken, was schon da ist «. Das heißt, alle die "Pfunde" sammeln, mit denen die Organisation PR-mäßig bereits wuchern kann! Wurde eine solche Liste erstellt (und vielleicht erstaunt festgestellt, was die Kolleginnen und Kollegen so alles anbieten und auf die Beine stellen!), gilt es, darauf aufbauend ein PR-Konzept und eine To-do-Liste zu erarbeiten, um die guten Inhalte und Angebote auch gezielt nach außen bekanntzumachen (vgl. Kasten »Checkliste PR-Konzept«). möchte Traditionen wahren und gleichzeitig die Jugendlichen kompetent für die Zukunft vorbereiten.

- Beschreibung der Zielgruppen: Wen wollen wir erreichen? Senioren? Junge Menschen? Menschen mit einer Behinderung? Kommunalpolitiker? Unternehmen? Nachbarn?

3. Strategie und Planung

- Planung der Maßnahmen nach Instrumenten: Welche PR-Instrumente setzen wir ein? Was wollen wir alles tun? (Website erstellen? Flyer? Pressearbeit?)

- Planung der Maßnahmen nach der Zeit: Was wollen wir wann tun? (Eine das Geschäftsjahr begleitende PR-Planung lässt sich leichter umsetzen und auf mehrere Schultern verteilen.)

- Planung der Maßnahmen nach dem Budget: Wie viel Geld brauchen wir (für Drucksachen u. a.) und wie können wir das bereitstellen (Haushalt, Förderer, Stiftungen)?

- Personalplanung: Wer macht die PR? Wer kann ihn unterstützen? Wahl der Präsentation: Wie stellen wir uns (nach innen und nach außen) dar? Welches Corporate Design (Logo. T-Shirts o. Ä.) wählen wir?

\section{Durchführung}

Die geplanten Bausteine der Strategie abarbeiten und umsetzen.

5. Kontrolle

Evaluation: Wie messen wir den Erfolg unserer Arbeit? Welches sind die Kriterien?

Martina Peters

\section{"Wir wollen in der Zeitung stehen!"}

Den Löwenanteil der Öffentlichkeitsarbeit macht nach wie vor die Pressearbeit aus - auch wenn die digitalen Medien und die Präsenz im Internet zunehmend wichtiger werden. Dabei ist die bedeutsamste Aufgabe einzuschätzen, wann Veranstaltungen und Projekte der Organisation interessant für die Redaktionen der Medien sind. Redakteurinnen und Redakteure brauchen Anlässe, also »Aufhänger «, um über soziale Neuigkeiten zu berichten. In der journalistischen Sprache sind das die Nachrichtenkriterien. Nachrichtenkriterien und damit "Aufhänger" können beispielsweise sein:
- lokaler Bezug (gilt für lokale Medien wie Tageszeitungen, lokale Hörfunkund Fernsehsender: Die Nachricht muss etwas mit der Stadt oder der Region zu tun haben.)

- Aktualität (neuer Vorstand tritt seinen Dienst an)

- Neuigkeit (erstes Angebot von Schulsozialarbeit in der Stadt)

- viele Betroffene (Träger bietet Stadtranderholung während der Sommerferien)

- Nutzwert (die Pflegedienstleiterin gibt ein Interview zur Pflege durch Angehörige)

- Fortschritt (die Werkstatt für behinderte Menschen öffnet ihren Versammlungsraum auch für Vereine in der Stadt)

- Kampf, Konflikt, Problem (Übergriffe auf Pflegepersonal nehmen zu)

- Prominenz (die Sozialministerin des Landes kommt zu Besuch)

- Folgenschwere (Treffen von Selbsthilfegruppen müssen ausfallen, da das Dach der Vereinshalle beim letzten Sturm eingebrochen ist.)

- Humor, Unterhaltsamkeit (Kindergarten gibt Sammlung mit Kinderaussprüchen heraus)

- Seltenheit (Organisation bietet als einzige der Stadt türkischsprachige Beratung an)

- Veranschaulichung eines aktuellen Themas (Interview mit Geschäftsführer zum Personalmangel im sozialen Bereich)

Aufgabe einer Organisation, die in die Zeitung kommen will, ist es, den Redaktionen solche Aufhänger mit diesen Nachrichtenkriterien anzubieten. Wie man sehen kann, gibt es wohl in jedem sozialen Unternehmen und in jedem Wohlfahrtsverband ausreichend davon!

\section{Was Öffentlichkeitsarbeit erfolgreich macht}

Bei der Umsetzung von Öffentlichkeitsarbeit sollten einige wichtige Regeln beachtet werden:

- "Man kann nicht nicht kommunizieren.«(Paul Watzlawick): Auch wenn eine Organisation sich der Presse verweigern will, kann daraus ein Zeitungsartikel entstehen (»Geschäftsführer war nicht zu einer Stellungnahme bereit«). 


\section{Fünf-Punkte-Sofortprogramm für eine bessere Öffentlichkeitsarbeit - ein Vorschlag}

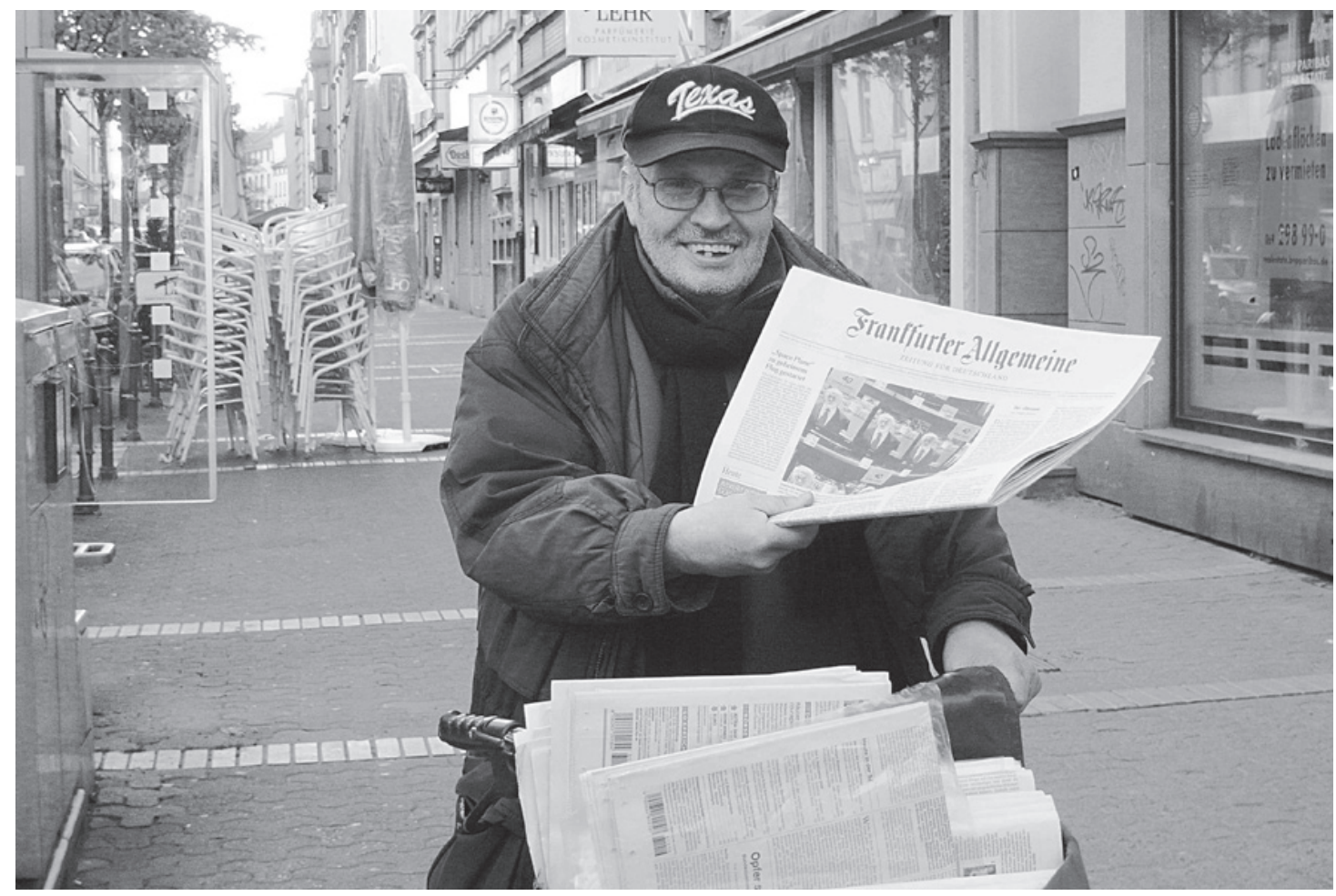

1 Konzeption: Beginnen Sie sofort mit den (Vor-) Arbeiten für - eine umfassende Konzeption der Öffentlichkeitsarbeit Ihrer Organisation. Sammeln Sie erstens internes und externes Material (Vereinssatzung, Protokolle von Mitgliederversammlungen, Vorstands- und Teamsitzungen, in denen es um Ziele der fachlichen Arbeit und um Öffentlichkeitsarbeit ging, Beispiel eigener und fremder Öffentlichkeitsarbeit). Erstellen Sie zweitens einen Zeitplan mit realistischen Etappen, bis wann was durch wen wie erledigt sein soll.

2 Dokumentation: Überlegen Sie, wie ein sinnvolles - das - heißt: ein ebenso wirkungsvolles wie einfaches - System zur Dokumentation der Arbeit Ihrer Organisation aussehen kann. Eine einfache Möglichkeit: Formulieren Sie in einem Tagebuch, einem Kalender oder einer EDV-Datei regelmäßig jeden Monat die drei oder fünf wichtigsten Ergebnisse oder Ereignisse in oder für Ihre Organisation.

3. Kontinuität: Legen Sie einen Timing-Kalender an, in dem 3. Sie für die Öffentlichkeitsarbeit Ihrer Organisation wichtige Ereignisse notieren. Das können Termine sein, die aus der Organi- sation selbst heraus wichtig sind (Jubiläen, neues konzeptionelles Angebot, Mitgliederversammlung, Vorstands- oder Mitarbeiterwechsel etc.) oder Entwicklungen, die von außen in Gang gesetzt werden (durch neue Gesetze, die städtische Haushaltsdebatte, Wahlen etc.).

1 Philosophie: Beginnen Sie mit der Formulierung der ein4. fachen und überzeugenden Antwort auf die Frage an Ihre Organisation: Wem bieten wir wie welchen Nutzen?

5 Innovation: Oft verbirgt sich hinter dem Wunsch nach 5. "mehr" oder "besserer" Öffentlichkeitsarbeit die uneingestandene Erkenntnis, dass mit der Organisation nicht mehr alles stimmt. Prüfen Sie, ob in Ihrer Organisation vor dem zweiten Schritt einer offensiven Öffentlichkeitsarbeit nicht zuerst ein Prozess der Neuorganisation und Neupositionierung angezeigt ist.

Quelle: Gerhard Pfannendörfer: Kommunikationsmanagement. Das ABC der Öffentlichkeitsarbeit für soziale Organisationen. Nomos Verlagsgesellschaft, Baden-Baden 1995. (Im Buchhandel vergriffen).
- Erfolgreiche Öffentlichkeitsarbeit muss kontinuierlich betrieben werden; also nicht nur einmal im Jahr, sondern in regelmäßigen Abständen immer wieder.

- Öffentlichkeitsarbeit produziert keine Erfolge von heute auf morgen; es wird wahrscheinlich nicht Artikel in allen örtlichen Zeitungen bei der ersten Aktion der Pressearbeit geben.

- Jeder Mitarbeitende ist ein "Botschafter" seiner Organisation; bewusst oder unbewusst. Jeder Mitarbeiter und jede Mitarbeiterin tragen dazu bei, ein Bild seiner Organisation in der Öffentlichkeit entstehen zu lassen.

- Medienvertreter sind wichtige Gesprächspartner für Unternehmen und Verbände; sie sollten deshalb schnell, regelmäßig, umfassend und zuverlässig informiert werden.

- Öffentlichkeitsarbeit ist Vertrauenssache; nur wer korrekt informiert, kann auf Dauer glaubwürdig sein.

- Nur wer gut informiert ist, kann gut informieren. Kenntnisse über die bundesweite Sozialwirtschaft und die Situation der Organisationen vor Ort sind nicht nur nützlich, sondern notwendig, um gute Öffentlichkeitsarbeit zu machen.

Und nicht vergessen: Klappern gehört zum Handwerk! Jeder Bericht in einer Fachzeitschrift, jeder Magazinbeitrag in einem örtlichen Fernsehsender kann ein Aufhänger für weitere Presse- und Öffentlichkeitsarbeit sein. 